\title{
Long-term, multidomain analyses to identify the breed and allelic effects in MSTN-edited pigs to overcome lameness and sustainably improve nutritional meat production
}

\author{
Ziyao $\mathrm{Fan}^{1 \dagger}$, Zhiguo $\mathrm{Liu}^{1 \dagger}$, Kui Xu ${ }^{1 \dagger}$, Tianwen $\mathrm{Wu}^{1}$, Jinxue Ruan ${ }^{1}$, Xinmin Zheng ${ }^{2}$, \\ Shideng Bao ${ }^{3}$, Yulian $\mathrm{Mu}^{1 *}$, Tad Sonstegard ${ }^{4 *} \& \mathrm{Kui}^{\mathrm{Li}^{1 *}}$ \\ ${ }^{1}$ State Key Laboratory of Animal Nutrition, Institute of Animal Sciences, Chinese Academy of Agricultural Sciences, Beijing 100193, China; \\ ${ }^{2}$ Hubei Key Laboratory of Animal Embryo Engineering and Molecular Breeding, Hubei Institute of Animal Science and Veterinary Medicine, \\ Hubei Academy of Agricultural Sciences, Wuhan 430064, China; \\ ${ }^{3}$ Department of Stem Cell Biology and Regenerative Medicine, Lerner Research Institute, Cleveland Clinic, Cleveland OH 44195, USA; \\ ${ }^{4}$ Acceligen Inc., Eagan MN 55121, USA
}

Received March 15, 2021; accepted April 29, 2021; published online June 4, 2021

\begin{abstract}
Beef and mutton production has been aided by breeding to integrate allelic diversity for myostatin (MSTN), but a lack of diversity in the MSTN germplasm has limited similar advances in pig farming. Moreover, insurmountable challenges with congenital lameness and a dearth of data about the impacts of feed conversion, reproduction, and meat quality in MSTN-edited pigs have also currently blocked progress. Here, in a largest-to-date evaluation of multiple $M S T N$-edited pig populations, we demonstrated a practical alternative edit-site-based solution that overcomes the major production obstacle of hindlimb weakness. We also provide long-term and multidomain datasets for multiple breeds that illustrate how MSTN-editing can sustainably increase the yields of breed-specific lean meat and the levels of desirable lipids without deleteriously affecting feed-conversion rates or litter size. Apart from establishing a new benchmark for the data scale and quality of genome-edited animal production, our study specifically illustrates how gene-editing site selection profoundly impacts the phenotypic outcomes in diverse genetic backgrounds.
\end{abstract}

MSTN-edited pigs, multidomain evaluation, genetic background, editing strategy

Citation: Fan, Z., Liu, Z., Xu, K., Wu, T., Ruan, J., Zheng, X., Bao, S., Mu, Y., Sonstegard, T., and Li, K. (2022). Long-term, multidomain analyses to identify the breed and allelic effects in $M S T N$-edited pigs to overcome lameness and sustainably improve nutritional meat production. Sci China Life Sci 65, 362-375. https://doi.org/10.1007/s11427-020-1927-9

\section{INTRODUCTION}

Sustainably increasing lean meat rates remains the primary economic breeding goal for modern food livestock (Fowler et al., 1976; Kanis et al., 2005; Merks et al., 2012). Generally, such animals have the highest ratio of greenhouse gas emission to unit protein produced (Havlík et al., 2014). On

$\uparrow$ Contributed equally to this work

*Corresponding authors (Yulian Mu, email: mouyulian@caas.cn; Tad Sonstegard, email: tad@acceligen.com; Kui Li, email: likui@caas.cn) the basis of existing genes that are known to promote lean meat yield, natural mutations in the myostatin (MSTN) gene have been broadly used in cattle and sheep breeding for over 50 years, and such mutations confer a remarkable increase in lean meat yield (Boman et al., 2010; Kambadur et al., 1997; McPherron and Lee, 1997). The mechanistic basis of such increases was unclear until a study was conducted in 1997 using knockout mice (McPherron et al., 1997). Follow-up experiments showed that this protein negatively regulates skeletal muscle cell proliferation and differentiation at pre- 
and postpartum stages, respectively (Joulia-Ekaza and Cabello, 2006; Lee and McPherron, 1999). Mutations in MSTN are associated with an increased skeletal muscle mass in humans and other mammals (Mosher et al., 2007; Schuelke et al., 2004; Yu et al., 2005). Breeding schemes exist to accelerate the genetic improvement of meat yield by increasing the frequency of inactivating mutations of MSTN in a population, but these have not been widely used due to the potential negative effects of some MSTN mutations on reproductive functions (i.e., calving ease and fertility) (Keele and Fahrenkrug, 2001).

In pigs, however, there are very few known natural MSTN mutations. The MSTN loci of the Large White (LW) and high-lean yielding Pietrain breeds were shown to contain a promoter affecting the temporal expression of MSTN (Stinckens et al., 2008; Yu et al., 2007). In 2019, a study described a nonsense single-nucleotide polymorphism (SNP) in exon 3 of MSTN of a particularly lean line of the LW breed (Matika et al., 2019). Although this SNP significantly increased lean meat production, severe hindlimb weakness was observed among $\mathrm{MSTN}^{-1}$ newborns (Matika et al., 2019). In as early as 2014, a similar phenotype was observed in major Western commercial breeds, including Landrace and LW, after gene targeting in exon 3 to disable the biological function of MSTN (Kang et al., 2014). This congenital hindlimb weakness defect is a prohibitive bottleneck to the safe and ethical application of MSTN-editing in pigs.

Although multiple studies have described the generation of various edits of the MSTN locus in pigs, the problem of hindlimb weakness in newborns remains unresolved in commercial breeds (Kang et al., 2014; Wang et al., 2015). Notably, no such hindlimb weakness was reported upon editing this locus in two local Chinese breeds (Qian et al., 2015; Wang et al., 2017). None of the studies on MSTNedited pigs to date have included more than three pigs; however, the true economic value of any trait in food animals is based on evaluations of resource populations and not just in proof-of-concept animals. Long-term and multidomain behavioral and production performance analyses can convincingly testify to the superiority of these animals before commercialization. None of the previous studies assessed long-term and comprehensively major commercial traits like feed conversion ratio, fertility, and meat nutritional quality (Kang et al., 2017; Kanis et al., 2005; Merks et al., 2012).

To overcome these challenges related to animal functionality and address the lack of robust performance data on commercial $\mathrm{MSTN}^{--}$pigs, we developed populations of Chinese indigenous high-fat Meishan (MS) and Western commercial high-lean LW pigs harboring edited alleles of the MSTN gene coding sequence (CDS). We comprehensively evaluated the performance and carcass traits of pigs from these populations to obtain a more precise statistical understanding of the effects of MSTN editing and to explore the need for diverse application strategies in different pig breeds. Our study details a successful strategy to remove any lethal hindlimb phenotype and provides insights into major production traits, including feed conversion, reproduction, meat quality, nutritional value, and increased lean meat yield in different breeds due to MSTN editing. Thus, our study addresses the key problems that have hindered the widespread application of MSTN editing in pigs to date and simultaneously highlights the strong commercial potential of this exciting technology in improving sustainability of animal protein production.

\section{RESULTS}

\section{A gene-editing strategy that prevents congenital hin- dlimb weakness in $\mathrm{MSTN}^{-1}$ piglets in Western com- mercial breeds}

To exploit the known ability of MSTN disruption in increasing lean meat yield, we explored multiple strategies to edit the MSTN locus in the genome of pigs with excellent traits. In the early stages of this study, TALENs (transcription activator-like (TAL) effector nucleases) were used to generate stop codon mutations in exon 3 of MSTN in cells derived from both LW and MS pig breeds. The target doublestrand (ds) DNA break site in exon 3 of MSTN was selected to replicate a naturally occurring deletion (11 bp) found in Belgian Blue cattle (Figure 1A), who are well known for their extremely heavy muscling phenotypes. An A/G base mutation following the 11-bp deletion was introduced into LW founder pigs (Figure 1A). In MS pigs, a 2-bp deletion within the 11-bp target deletion was introduced (Figure 1A). The edits for both LW and MS pigs were designed to cause frameshift mutations that would produce a premature termination of the C-terminal region of the MSTN protein.

The MSTN-edited populations were created using the traditional linebreeding method (Figures S1 and S2 in Supporting Information). Since its initiation in 2012, our MSTNedited pigs are now in their eighth generation and number $>1,000$. However, all of the $\mathrm{MSTN}^{-1-} \mathrm{LW}$ piglets with exon 3 mutations showed hindlimb weakness and only survived for 1-2 days (Figure 1B). All $M_{S T N^{-1}}$ piglets were provided with as much special care as possible, including bottlefeeding alone in an enclosure where the piglets and sow were in sight of each other. Any piglet exhibiting significant suffering was euthanized using pentobarbital. After the accuracy of genome editing and irreversibility of the phenotype had been confirmed, $M S T N^{-1-}$ LW pigs were not included in further breeding plans. In contrast, all of the $\mathrm{MSTN}^{-1-} \mathrm{MS}$ pigs ( $N=216$; total five generations) with a similar exon 3 mutations had normal posture and mobility (Figure 1C).

Histological comparison of the biceps femoris showed that the number of nuclei was significantly increased and skeletal 


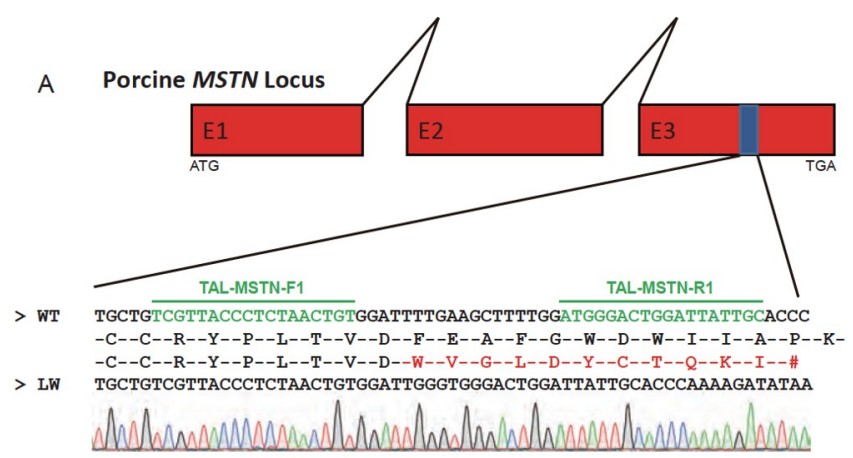

WT TGCTGTCGTTACCCTCTAACTGTGGATTTTGAAGCTTTTGGATGGGACTGGATTATTGCACCC -C--C--R--Y--P--L--T--V--D--C--C--R--Y--P--L--T--V--D--F--D--F--W--M--G--L--D--Y--C--T--O--K--I--\#

$>$ MS
B

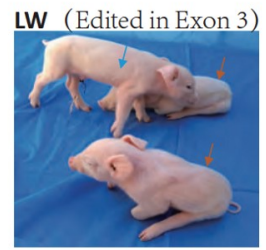

c

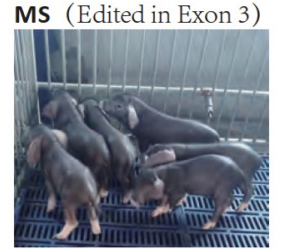

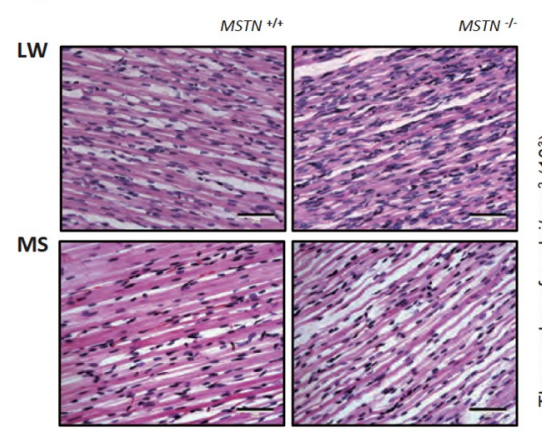

$\mathrm{F}$

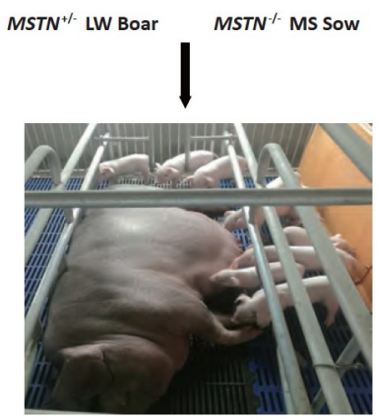

G

LW: CTCtAACtGTGGATTGGGTGGGATGGATtATtGCACCC MS:
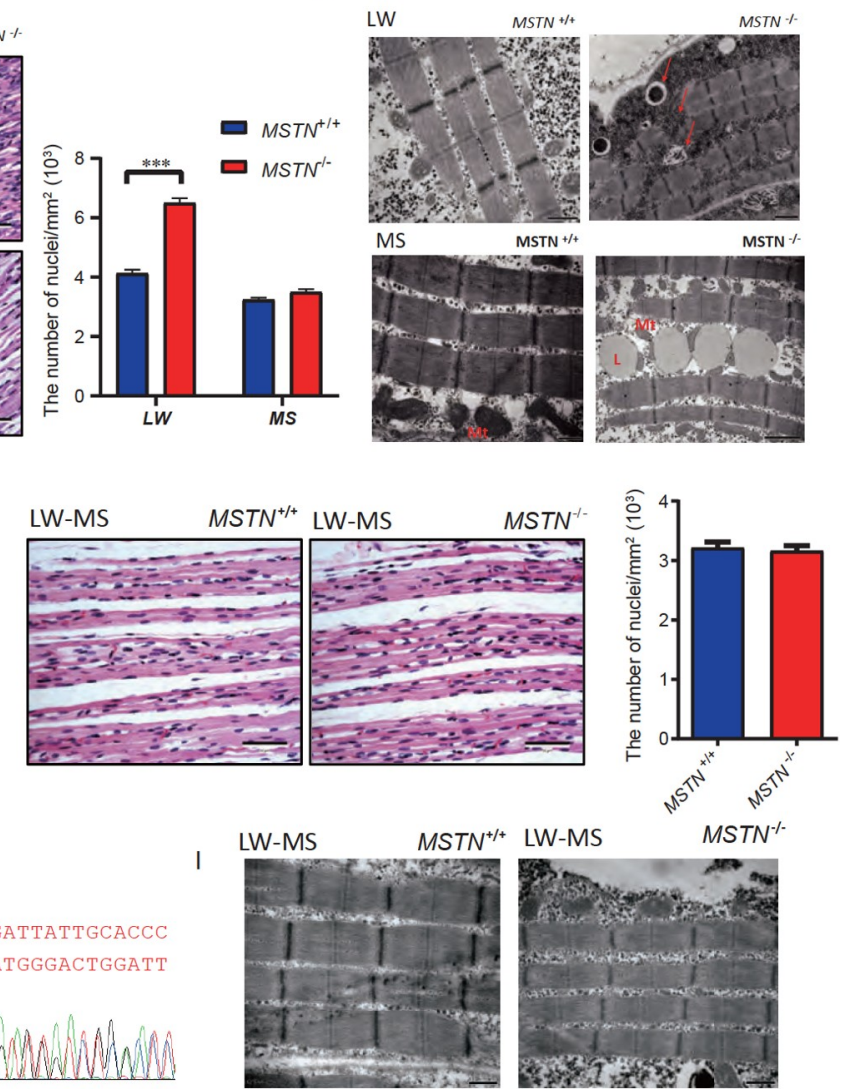

Figure 1 The impact of breed on hindlimb weakness in MSTN-edited piglets. A, The editing strategy for exon 3 of the MSTN locus in Chinese Meishan (MS) and Western commercial Large White (LW) pigs using TALEN. DNA sequencing showed a base mutation (A/G) following an 11-bp deletion, the same as that in Belgian Blue cattle, in exon 3 in the LW founder pigs, whereas in the MS founder pigs, a 2-bp sequence was deleted within this 11-bp sequence. The green sequence is the TAL recognition sequence. The red sequence represents the change in the amino acid sequence by frameshift mutation. The gene edit in the exon 3 of the MSTN locus in LW and MS genomes leads to frameshift mutation and premature termination (the new stop codon represents the terminating protein translation site generated by gene editing). B, $M S T N^{-1}$ LW piglets (deletion in exon 3) showed severe hindlimb weakness (red arrows, $M S T N^{-1}$ piglets; blue arrow, $M S T N^{+/-}$littermates). C, $M S T N$-edited $M S T N^{-1-}$ MS piglets (deletion in exon 3) were able to stand normally. D, H\&E staining showed a large number of gathered nuclei and broken muscle fibers in the biceps femoris of $M S T N^{-1}$ LW pigs. Scale bars $=50 \mu \mathrm{m}$. The number of nuclei in the section was assessed using Student's $t$-tests. ***, $P<0.001$; not significant: $P>0.05$, not labeled. E, Transmission electron microscopy (TEM) of the biceps femoris of $M S T N^{-1-}$ and WT LW pigs: Mt, mitochondria; L, lipid droplets; the arrows indicate myofibril rupture. Scale bars $=1 \mu \mathrm{m}$. F, The $M S T N$-edited $M S T N^{-1-}$ LWMS offspring ( $N=16)$ could stand and run normally. G, Sequencing of the edited sites of $M S T N^{-1}$ LW-MS hybrid pigs. H, H\&E staining of the biceps femoris of WT and $M S T N^{-1-}$ LW-MS hybrid pigs. Scale bars $=50 \mu \mathrm{m}$. The number of nuclei in the section was assessed using Student's t-tests, not significant: $P>0.05$, not labeled. I, TEM of the biceps femoris of WT and $M S T N^{-1-}$ LW-MS hybrid pigs. Scale bars $=1 \mu \mathrm{m}$. 
muscle fibers were partially fractured in the $\mathrm{MSTN}^{-1-} \mathrm{LW}$ pigs, compared with the $M S T N^{-1-}$ MS and wild-type (WT) LW animals (Figure 1D). Transmission electron microscopy (TEM) further revealed that large number of inclusion bodies had invaded the broken myofibrils and accumulated in the biceps femoris of the $M S T N^{-1}$ LW pigs, whereas no such bodies were detected in $\mathrm{MSTN}^{-1-}$ MS pigs. Moreover, compared with WT MS pigs, lipid droplets surrounded by mitochondria between myofibrils were also increased in $\mathrm{MSTN}^{-1-}$ MS pigs (Figure 1E). Notably, all of the offspring resulting from crosses between the two breeds (i.e., hybrid $\mathrm{MSTN}^{-/}$LW-MS offspring) could stand and run normally (Figure $1 \mathrm{~F}$ and G). Furthermore, hematoxylin and eosin (H\&E) staining and TEM analyses did not find any obvious pathological features, such as inclusion bodies, a significant increase in the number of nuclei, and broken myofibrils, in the biceps femoris of $\mathrm{MSTN}^{-/-}$ LW-MS pigs (Figure $1 \mathrm{H}$ and I).

To specifically overcome hindlimb weakness in purebred LW animals, we initially confirmed that exon 3-edited $M S T N$ mRNA can be transcribed (Figure 2A and Data S1 in Supporting Information). Subsequently, Western blotting revealed that the size of the protein translated from the exon 3-edited MSTN transcripts was, as expected, smaller than that from the WT full-length MSTN (375 aa); specifically, the mutated MSTN in MS and LW pigs were 303 and 300 aa long, respectively. The functional consequence of this relates to a critical amino acid, C339, which is required for MSTN dimerization (Cotton et al., 2018; Walker et al., 2017). The translation products of truncated MSTN do not include $\mathrm{C} 339$, and the edited MSTN proteins could not form dimers (Figure 2B). This is a significant outcome of our study because previous studies reported that MSTN can only be secreted from cells in a dimerized form (Cotton et al., 2018).

To pursue the idea about disrupted protein secretion, we detected the expression of marker genes for ER stress in the skeletal muscle of $M S T N$-edited pigs, including glucose regulated protein 94 (GRP94), activating transcription factor 3 (ATF3), and heat shock protein family A (Hsp70) member 5 (HSPA5 GRP78/BiP), and found that their expression in the skeletal muscles of $M_{S T N^{-1}}$ LW pigs was significantly higher than that of WT LW and $M S T N^{-1-}$ MS pigs (Figure 2C). We also detected the phosphorylated (activated) form of eukaryotic translation initiation factor 2A (eIF2A) (Park et al., 2006) in the skeletal muscles of $M S T N^{-1}$ LW pigs but not in those of WT LW and $M S T N^{-1}$ MS pigs, indicating ER stress in the skeletal muscles of $M S T N^{-/}$LW pigs (Figure 2D).

We eventually overcame the hindlimb pathology issues in LW pigs by editing a completely different region of MSTN that avoided any issues with the dimerization and export of the protein product. Specifically, we chose editing sites near the $5^{\prime}$-terminal end of MSTN CDS. CRISPR/Cas9 editing with the designed small guide RNAs (sgRNAs) resulted in the successful deletion of $40 \mathrm{bp}$ from exon 1 (Figure 2E). All $M S T N^{-1-}$ LW offspring having the MSTN exon 1 edit were able to move with ease (Figure 2F), and no abnormalities in the biceps femoris were detected on H\&E and TEM analyses (Figure $2 \mathrm{G}$ and $\mathrm{H}$ ). Extraordinarily, when we introduced this exon 1 edit into the pure Landrace breed, all of the $M_{S T N}{ }^{--}$ offspring could stand normally, clearly suggesting that this exon 1 edit can overcome the issue of lameness in many breeds that has limited the utility of $M S T N$-based genetic modifications.

\section{MSTN-editing leads to similar feed conversion due to reduced exercise energy consumption but increased maintenance energy consumption}

As observed by video-based monitoring of the MSTN-edited MS pigs over time, these pigs can be accurately described as "lazy but muscular"; in contrast, the WT animals are less muscular but substantially more likely to move around and exercise. We assessed the health of the pigs via blood cell count and serum content analyses and found no significant differences (Table S1 in Supporting Information).

To quantify the apparently reduced duration and frequency of physical activity in the MSTN-edited MS pigs, we analyzed the behavior of four $M S T N^{-1}$ and four WT pigs via video recordings. The WT pigs showed $>30 \mathrm{~min}$ of active movement per day than the $M S T N$-edited pigs $(P<0.01$, Figure $3 \mathrm{~A}$ ). Our experiments were conducted using a precision feeding system, which included electronic ear-tag identification, trough feeding and weighing, as well as individual real-time weight measuring. Moreover, only one pig was allowed to enter the limit box at a time to ensure the continuous and accurate recording of the free-feed intake of each individual under group feeding conditions and directly calculating the feed-conversion ratio (FCR). Although the significant difference in movement implies potentially reduced energy requirements, the FCR (Figure 3B) did not differ between the $M S T N$-edited and WT MS pigs in both males and females (3.97 of WT female $(N=20), 3.92$ of $M S T N^{-1-}$ female $(N=20) ; P=0.0725$ and 3.95 of WT male $(N=20), 3.92$ of $M_{S T N^{-1}}$ male $\left.(N=20) ; P=0.2854\right)$. Moreover, no difference was between the $M S T N$-edited and WT LW pigs (2.54 of WT female $(N=24), 2.55$ of $M S T N^{+/}$female $(N=24) ; P=0.7356$ and 2.55 of WT male $(N=24), 2.56$ of $M_{S T N}^{+-}$male $\left.(N=24) ; P=0.7671\right)$.

Next, to measure the energy expenditure of pigs, we focused on differences in the maintenance net energy between $M S T N^{-1-}$ and WT MS pigs in fasting and resting states (from 10:00 pm to 6:00 am) using a net energy measurement device, which is a completely enclosed chamber for the realtime monitoring of atmospheric $\mathrm{O}_{2}$ and $\mathrm{CO}_{2}$ levels. The pigs 

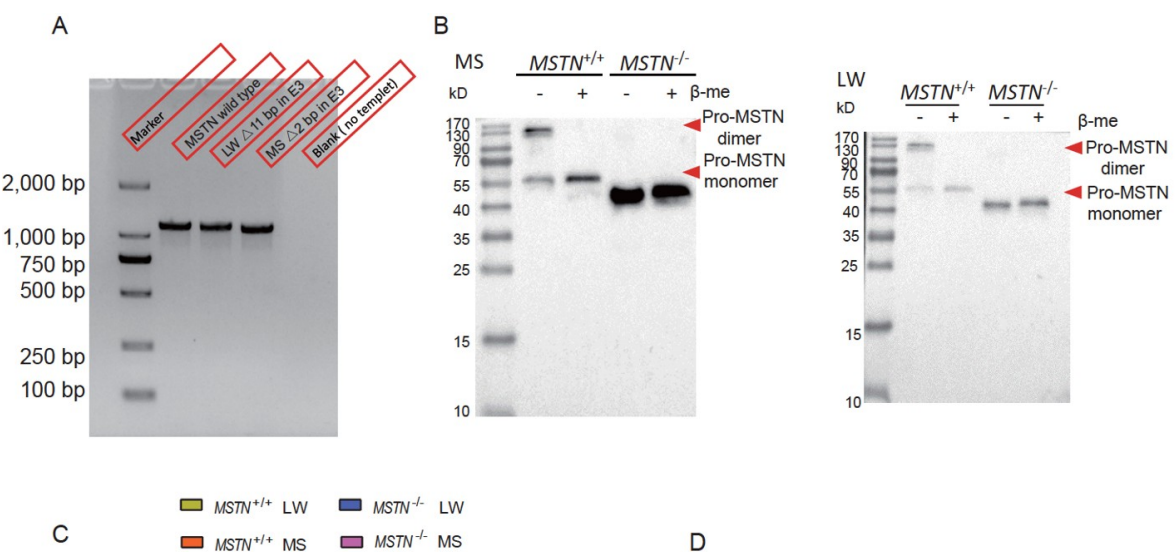

D
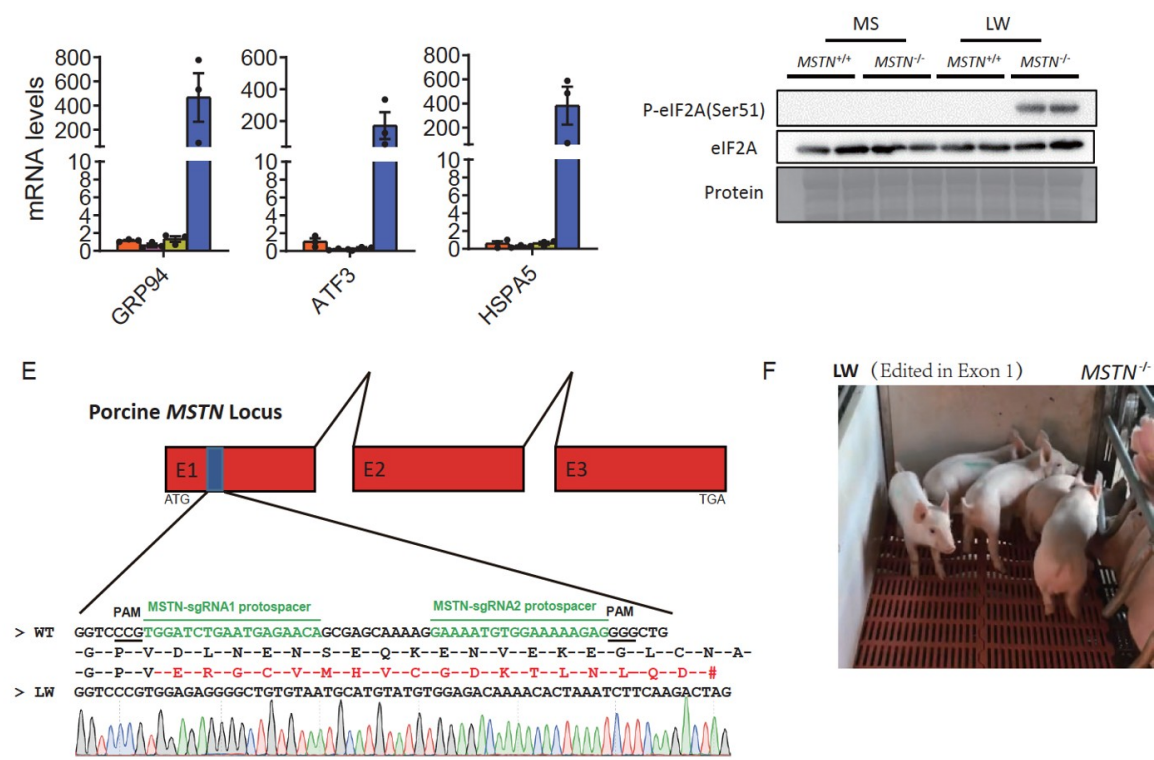

G

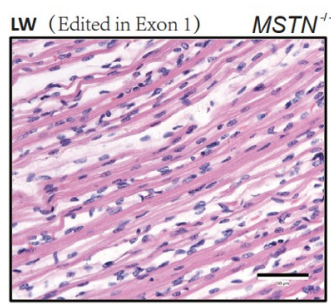

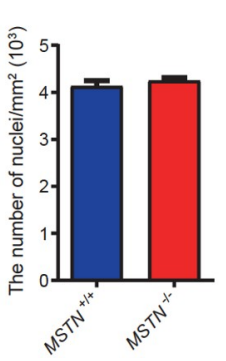

$\mathrm{H}$

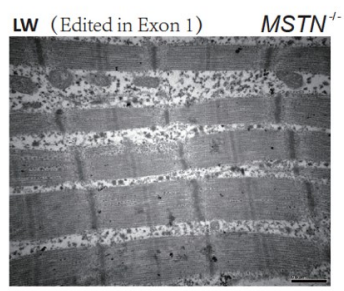

Figure 2 Alternative edit-site-based solution avoids ER stress and overcomes the hindlimb weakness. A, Reverse transcription polymerase chain reaction for the whole length of MSTN CDS showed that the mutated MSTN locus can be transcribed. B, Western blotting revealed that these transcripts produced slightly smaller peptides (mutated MSTN in MS pigs, 303 aa; mutated MSTN in LW pigs, 300 aa) than the normal MSTN protein (375 aa), and that the peptides from the edited transcripts could not form MSTN dimers. $\beta$-me represents mercaptoethanol used in the protein-loading buffer to destroy disulfide bonds for forming dimers. C, The expression of ER stress marker genes in the skeletal muscle of MSTN-edited pigs, including GRP94, ATF3, and HSPA5 $(N=3)$. D, Western blotting for the eIF2a protein and phosphorylated eIF2a (at Ser51) in the skeletal muscles of MSTN-edited LW and MS pigs. E, The editing strategy of exon 1 of MSTN in LW pigs using CRISPR/Cas9. DNA sequencing showed a biallelic $40 \mathrm{bp}$ deletion in MSTN of the piglets. The green sequence represents the sgRNA protospacer and the PAM sequence is underlined. The gene editing in exon 1 of the MSTN locus leads to a code-shift mutation and premature termination (new stop codon represents the terminating protein translation site generated by gene editing). The red sequence represents the changed amino acid sequence resulting from the code-shift mutation. F, $M S T N^{-1-} \mathrm{LW}$ piglets (deletion in exon 1) had no hindlimb weakness and could move with ease. $\mathrm{G}, \mathrm{H} \& \mathrm{E}$ staining of the biceps femoris of $M S T N^{-1} \mathrm{LW}$ piglets (deletion in exon 1) detected no abnormalities. Scale bars $=50 \mu \mathrm{m}$. The number of nuclei in the section was assessed using Student's $t$-tests. The WT LW piglets shown in Figure 1D were used as a control, not significant: $P>0.05$, not labeled. H, Transmission electron microscopy of the biceps femoris of $M S T N^{-1}$ LW piglets (deletion in exon 1), Scale bars=1 $\mu \mathrm{m}$.

exhibited limited movement in the chamber along with increased heat production (HP) (Figure 3C), revealing that the maintenance energy consumption of the $M S T N$-edited pigs was significantly increased compared with that of the WT 

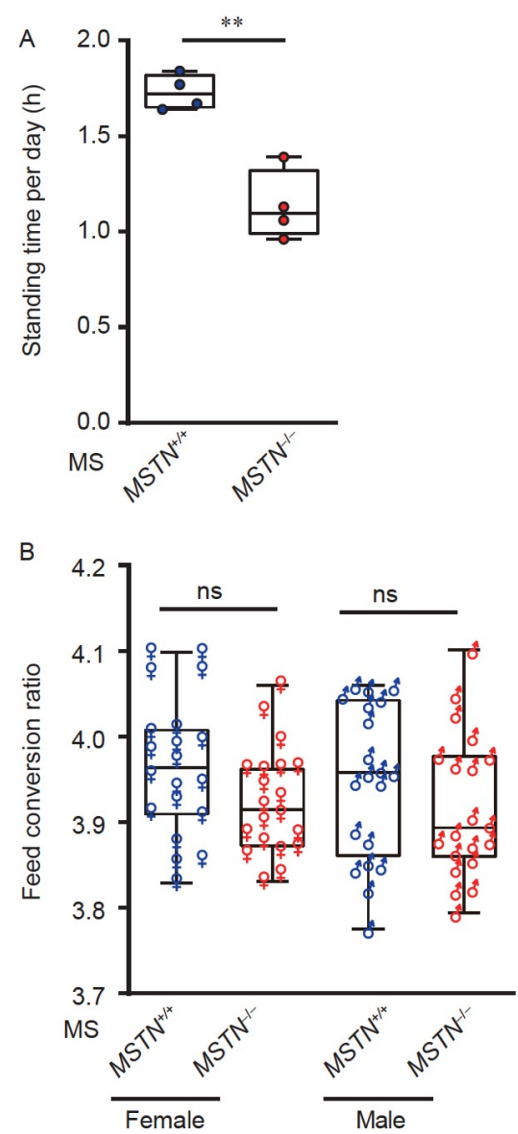

C
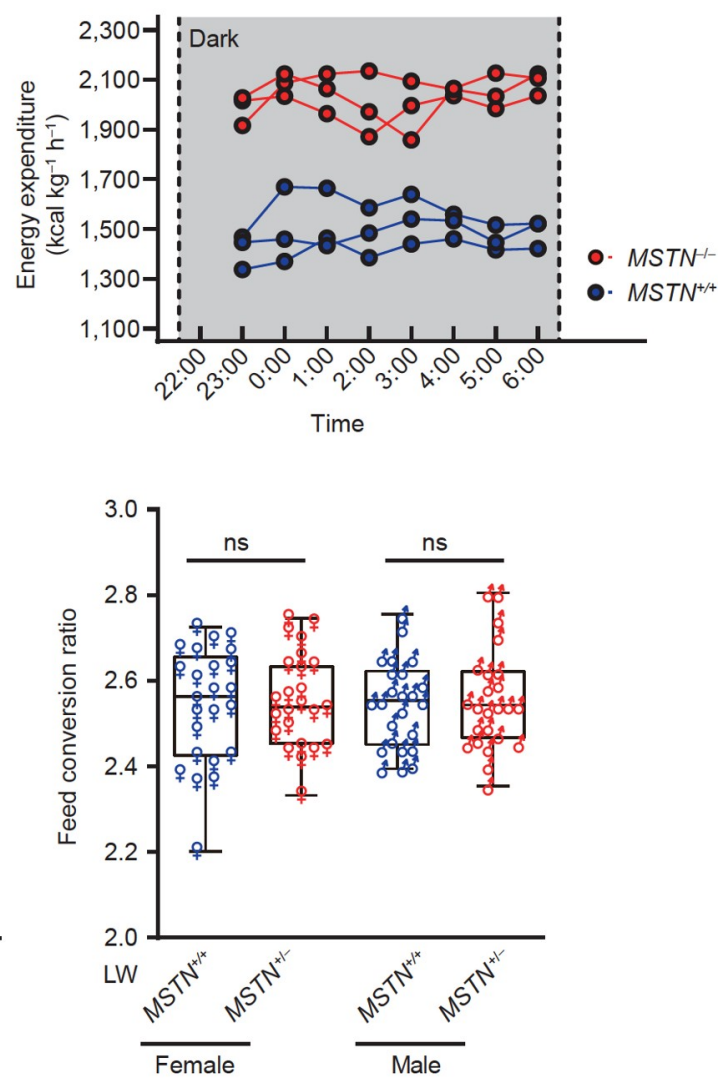

Figure 3 Similar feed-conversion ratio results from the decreased movement and increased thermogenesis. A, Average standing time of $M S T N^{-1}(N=4)$ and WT MS pigs $(N=4)$ per day. B, Feed conversion of $M S T N^{-1-}$ and WT in LW and MS. C, Heat production of $M S T N^{-1-}$ and WT MS pigs under absolute resting and fasting conditions. Inferential statistical significance was assessed using Student's $t$-tests throughout the study. In the box plot, the middle line of each box is the median, the boxes are the width of the interquartile range (IQR), and the whiskers extend to the farthest observation still less than 1.5 times the IQR; all points are plotted for clarity. *, $P<0.05$; **, $P<0.01$; **, $P<0.001$; ns, not significant, $P>0.05$.

pigs. Thus, although the $M S T N$-edited pigs have reduced exercise energy consumption, they have significantly higher energy consumption rates for their physiological maintenance, which accounted for our observation that their overall FCR was not different from that of the WT pigs.

\section{No dystocia or significant changes in the litter size of MSTN-edited sows}

Reproductive performance, particularly litter size, is the most important performance and breeding target for sows in pig breeding (Kanis et al., 2005). In light of reports about the increased costs and high incidence of dystocia due to narrow birth canal and increasing birth weight of Belgian blue cattle (Kolkman et al., 2010; Sutherland, 1985), we carefully examined the effect of $M S T N$-editing on the reproductive performance of sows in our population $\left(M_{S T N}^{-/}\right.$and $M S T N^{+/-}$MS sows and $M S T N^{+/-}$LW sows). No dystocia was observed in the MSTN-edited MS or LW sows. Moreover, the total litter sizes of the MSTN-edited sows did not differ from those of the WT sows (Figure 4A-D). The birth weights of the MSTN-edited piglets did not differ from those of the WT piglets, which could potentially explain the absence of dystocia. Finally, to detect lactation performance, we calculated the raising rate (weanling piglets/live piglets born) and found no significant differences in lactation performance between the MSTN-edited and WT sows (Figure S3 in Supporting Information).

\section{$M S T N$-editing increased lean meat yield in both MS and LW pigs but with distinct modes}

We worked with the national performance testing centers in China to evaluate the effect of $M S T N$-editing on the lean meat production performance of the Chinese high-fat breed (MS) and the commercial lean breed (LW) relative to their WT littermates. For both breeds, WT, MSTN ${ }^{+/-}$, and $M S T N^{-1-}$ pigs were evaluated. For MS pigs assessed at 6 months of age, the dressing percentages were not significantly different among the three genotypes (Tables S2 and S3 in Supporting Information). Compared with the change in the production of lean meat between WT and $M_{S T N}{ }^{+/}$pigs $(47.34 \%$ vs. 

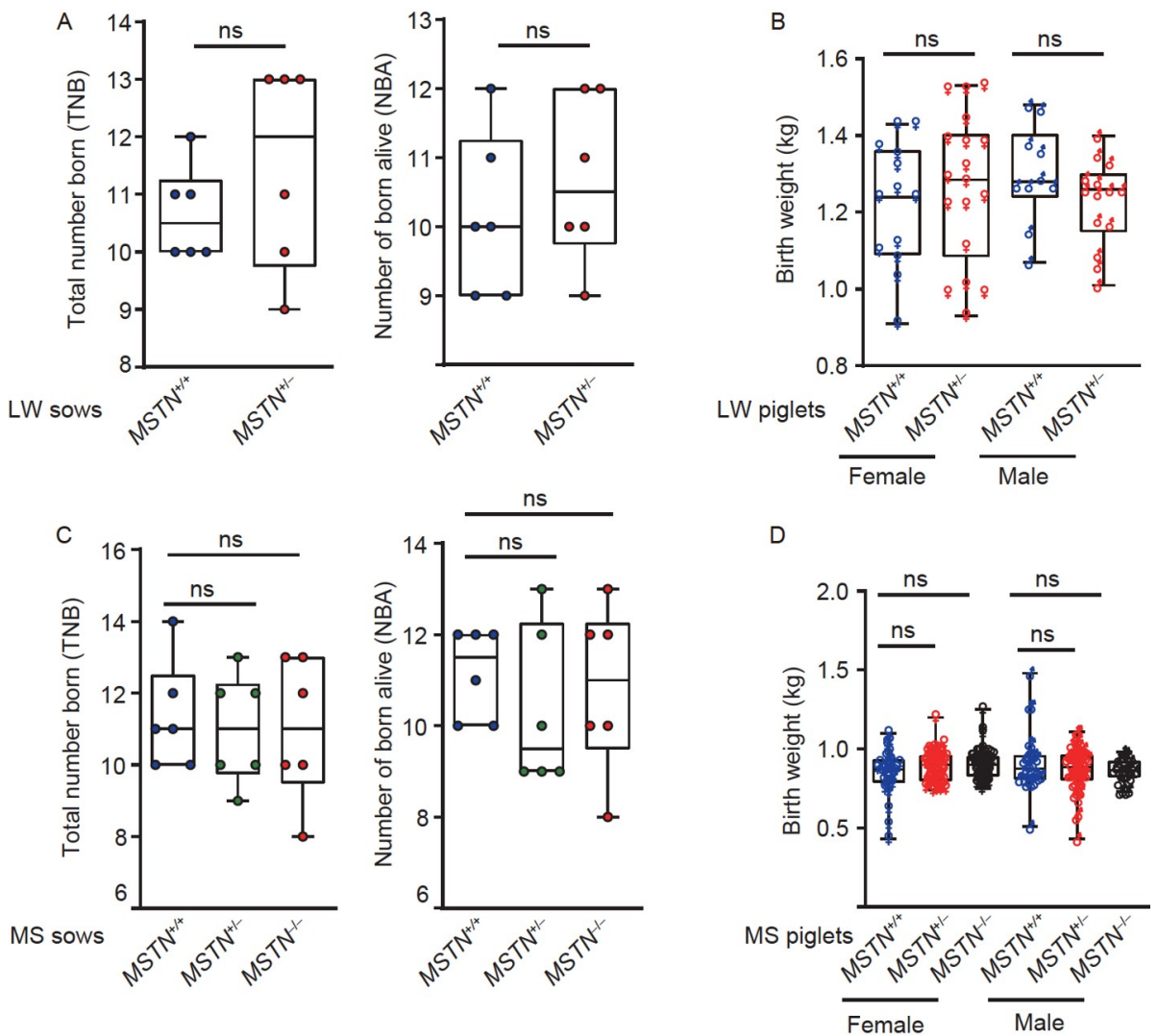

Figure 4 No dystocia or significant effects on the maternal reproductive traits of $M S T N$-edited sows. A, Total number born (TNB) and the number born alive (NBA) for $M_{S T N}{ }^{+/}(N=6)$ and WT $(N=6)$ LW sows. B, The birth weight of $M S T N$-edited and WT LW piglets, including $M S T N^{+/+}$female $(N=13)$, $M S T N^{+/-}$female $(N=18), M S T N^{+/+}$male $(N=10)$, and $M S T N^{+/-}$male $(N=14)$. C, TNB and NBA data for $M S T N$-edited and WT MS sows, including $M S T N^{+/+}$ $(N=6), M_{S T N}^{+/}(N=6)$, and $M S T N^{-/}(N=6)$. D, The birth weight of $M S T N$-edited and WT MS piglets, including $M S T N^{+/+}$female $(N=29), M S T N^{+/}$female $(N=56), M S T N^{-\alpha}$ female $(N=51), M_{S T N^{+/+}}$male $(N=25), M_{S T N^{+/-}}$male $(N=50)$, and $M S T N^{-1-}$ male $(N=24)$. ns, not significant, $P>0.05$.

$50.39 \% ; P=0.0299)$, there was a huge and significant increase in the amount of lean meat produced by the $M S T N^{-1-}$ pigs $(73.85 \% ; P<0.0001)$. The observed relative increase in lean meat was $56 \%$ more in $M S T N^{-1-}$ MS than in WT MS pigs. There was no significant difference in the amount of fat meat between the WT and $M S T N^{+/-}$pigs $(26.31 \%$ vs. $22.80 \% ; P=0.0743)$; the $M S T N^{--}$pigs had only $6.12 \%$ fat meat $(P<0.0001)$, which is a significant relative decrease of $76.74 \%$.

Recalling that the exon 3 TALEN-edited $M S T N^{-1}$ pigs could not stand, the $M S T N^{+/}$LW pigs underwent exon 3 editing whereas the $M S T N^{-1}$ LW pigs underwent exon 1 editing; the WT pigs in this comparison were littermates of the exon 3 edit. Carcass performance was assessed at 6 months of age, and the dressing percentages were not significantly different among the three genotypes. However, the lean meat production of both the $\operatorname{MSTN}^{+/-}(N=14)$ and the $M S T N^{-1-}$ pigs $(N=4)$ were significantly higher than that of the WT $(N=19 ; 69.44 \%)$, with increases of $8.73 \%(75.50 \%$; $P<0.0001)$ and $9.92 \%(76.33 \% ; P<0.0001)$, respectively. Conversely, the fat meat rates of both $M S T N^{+-}$pigs $(N=6)$ and $\mathrm{MSTN}^{--}$pigs $(N=4)$ were significantly decreased com- pared with that of the WT $(N=6 ; 10.36 \%)$ by $50.70 \%(5.11 \%$; $P=0.0022)$ and $54.19 \%(4.75 \% ; P=0.0204)$, respectively (Table 1).

The comparisons of the MSTN-editing-mediated improvements in lean meat production in the MS and LW breeds highlight two phenomena. First, although the difference in lean meat rates between the WT of the two breeds is large, the highest level of lean meat for $M_{S T N^{--}}$pigs of both breeds plateaued between $73.85 \%$ and $76.33 \%$; this level appears to be the maximum rate achievable. Second, there is an apparent difference in the manner by which the $M S T N^{+/}$ and $M S T N^{-1-}$ MS pigs differed from the LW pigs. In MS pigs, the lean meat rate of the $M S T N^{+/}$pigs was slightly higher than that of the WT, whereas that of the $M S T N^{-1-}$ pigs was significantly increased compared with that of the $M_{S T N}{ }^{+/}$ pigs. In contrast, for LW pigs, the lean meat rate of the $M S T N^{+/-}$pigs was much higher than that of the WT, whereas that of the $\mathrm{MSTN}^{-1}$ pigs was slightly increased compared with that of the $\mathrm{MSTN}^{+/-}$pigs (Figure 5A). Regarding the fat rate, the $\mathrm{MSTN}^{+/-}$and $M S T N^{-1-}$ MS and LW pigs also showed apparently distinct modes but with a trend opposite to that observed for lean meat production (Figure 5B). 
Table 1 Lean meat rate and fat rate of MSTN-edited MS and LW pigs ${ }^{\text {a) }}$

\begin{tabular}{|c|c|c|c|c|c|c|}
\hline & \multirow{2}{*}{ Breed } & \multirow{2}{*}{$\begin{array}{c}M_{S T N}^{+/+} \\
\text {Mean } \pm \text { SEM }\end{array}$} & \multicolumn{2}{|c|}{$\operatorname{MSTN}^{+/-}$} & \multicolumn{2}{|c|}{$\operatorname{MSTN}^{-1-}$} \\
\hline & & & Mean \pm SEM & vs. WT & Mean \pm SEM & vs. WT \\
\hline \multirow{2}{*}{ Lean rate $(\%)$} & MS & $47.34 \pm 0.7575 N=16$ & $50.39 \pm 1.150 N=12$ & $*$ & $73.85 \pm 0.5057 N=12$ & $* * *$ \\
\hline & LW & $69.44 \pm 0.3986 N=19$ & $75.50 \pm 0.3480 N=14$ & $* * *$ & $76.33 \pm 0.4795 N=4$ & $* * *$ \\
\hline \multirow{2}{*}{ Fat rate $(\%)$} & MS & $26.31 \pm 0.9622 N=16$ & $22.80 \pm 1.763 N=12$ & ns & $6.123 \pm 0.4939 N=12$ & $* * *$ \\
\hline & LW & $10.36 \pm 1.165 N=6$ & $5.108 \pm 0.5356 N=6$ & ** & $4.746 \pm 1.633 N=4$ & * \\
\hline
\end{tabular}

a) Significance was calculated using Student's $t$-test. *, $P<0.05$; **, $P<0.01$; ***, $P<0.001$; ns, not significant, $P>0.05$.
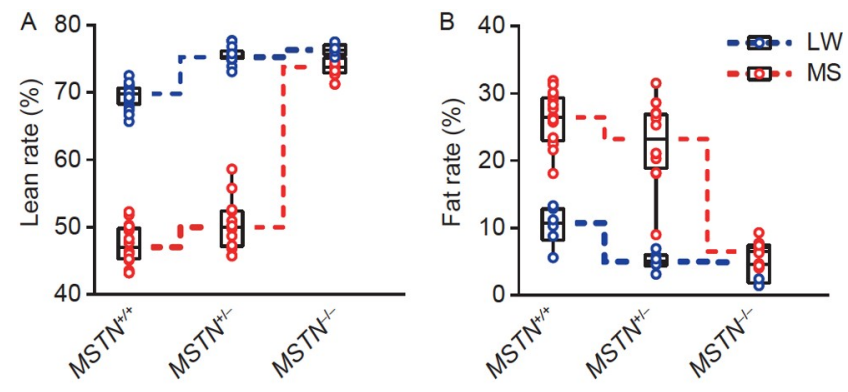

Figure $5 M S T N$-editing leads to different trends of change for lean and fat rates of MS (Chinese high-fat) and LW (Western high-lean) pigs. The carcass traits of MSTN-gene-edited and WT pigs (carcass age, 6 months) determined by third-party national performance testing centers of China. A, Comparison of the increasing trend in lean meat production induced by $M S T N$-editing between MS and LW pigs. B, Comparison of the decreasing trend in fat rate induced by $M S T N$-editing between MS and LW pigs.

\section{MSTN-edited pork has better tenderness, higher protein, lower fat content, and increased levels of poly- unsaturated fatty acids}

Longissimus dorsi samples were collected from the MS and LW pigs of all three genotypes to evaluate the effect of $M S T N$-editing on indicators of pork quality, such as $\mathrm{pH}$, color, drip loss, and shear force. We found significantly decreased shear force in all MSTN-edited pork of the LW $\left(74.76 \mathrm{~N}\right.$ of WT $(N=4) ; 46.41 \mathrm{~N}$ of $\operatorname{MSTN}^{+/-}(N=6)$, $P=0.0044 ; 44.40 \mathrm{~N}$ of $\left.\mathrm{MSTN}^{-1-}(N=4), P=0.0054\right)$ and MS pigs $\left(80.05 \mathrm{~N}\right.$ of WT $(N=18) ; 65.33 \mathrm{~N}$ of $M S T N^{+/-}(N=12)$, $P=0.1011 ; 42.73 \mathrm{~N}$ of $\left.M_{S T N}{ }^{-/}(N=13), P<0.0001\right)$. These results collectively suggest that $M S T N$-edited pork is likely to be substantially tenderer than WT pork (Figure 6A). Moreover, there was a clear difference in the color of the pork for both breeds, with the $\mathrm{MSTN}^{-1}$ pork being more whitish. Meat color scores for LW pork were 3.47 for WT, 3.32 for $\operatorname{MSTN}^{+-}(P=0.4931)$, and 2.63 for $M_{S T N}{ }^{-/}$ ( $P=0.0169)$, whereas the scores for MS pork were 4.06 for WT, 3.75 for $\operatorname{MSTN}^{+/-}(P=0.0219)$, and 3.46 for $M_{S T N}{ }^{--}$ $(P<0.0001)$ (Figure 6B). The meat quality performance parameters of all individuals covering the two breeds and three MSTN genotypes were within the range of normal meat quality (Figure 6C and D; also summarized in Tables S2 and $\mathrm{S} 3$ in Supporting Information).

Next, we focused on the nutritional quality of MSTN-edi- ted pork. As expected based on the observed lean meat phenotypes, amino acid content analysis showed that the majority of the amino acids in the MSTN-edited LW pork $\left(M S T N^{-/}, N=4\right.$ and $\left.M S T N^{+/-}, N=9\right)$ were present at significantly higher levels than in the WT pork $(N=12)$; only glycine, histidine and tyrosine did not accumulate at significantly higher levels (Table S4 in Supporting Information). Compared with those in the WT pork $(N=10)$, the levels of polyunsaturated fatty acids (PUFA) - including both the n-6 and n-3 families of essential PUFAs - were enriched in the $M S T N$-edited LW lean meat $\left(M S T N^{-1}, N=4\right.$ and $M S T N^{+/}, N=10$; Figure $\left.7 \mathrm{~A}-\mathrm{C}\right)$. Note that although there were more PUFAs in the MSTN-edited pork, the distribution, i.e., n-6/n-3 ratio, of the PUFAs did not differ between the $M S T N^{+/-}$and WT pork, but was significantly reduced in $\mathrm{MSTN}^{-1-}$ pork (Figure 7D; Table S5 in Supporting Information). Furthermore, a similar increase in the overall PUFA levels was observed in the adipose tissues, including in subcutaneous fat (Table S6 in Supporting Information). Collectively, these results indicate that $M S T N$-edited pork is likely beneficial to human health, particularly for people with cardiovascular diseases.

\section{DISCUSSION}

Strategies centered on MSTN-editing have been widely considered a promising single gene edit approach for accelerating the breeding progress to improve meat production in pigs. However, the congenital defect phenotypes resulting from $M S T N$-editing that have been repeatedly reported for $\mathrm{MSTN}^{-1}$ commercial pig breeds and a lack of data on production performance traits, carcass traits, and nutrition have all lowered prospects for future commercialization of $M S T N$ edited pigs (Kang et al., 2014; Wang et al., 2015). The longterm multigenetic background and extensive multidomain performance evaluations generated in our study go a long way in reducing these concerns. Indeed, our study showed that the major problem of hindlimb weakness can be overcome by adopting alternative edit sites, and both our sustainability of carcass performance and compelling nutritional data make the prospect of $M S T N$-edited pigs even more attractive than they were previously. 

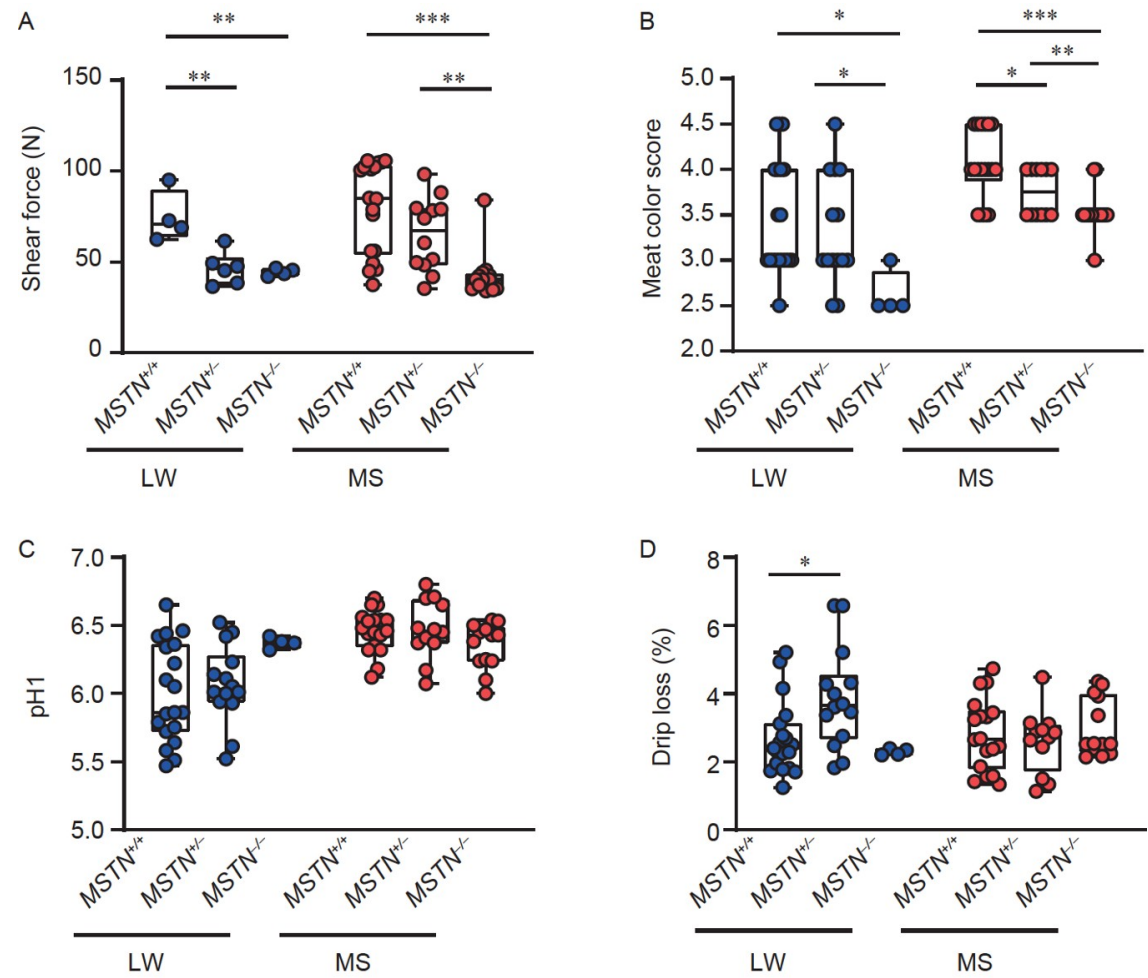

Figure 6 Comparison of meat quality among the three MSTN genotypes of two breeds. Meat was collected from MSTN-edited and WT pigs (MS carcass weight at 6 months $(\sim 50 \mathrm{~kg})$ or LW carcass age at 6 months $(\sim 120 \mathrm{~kg}))$ and meat quality was determined by third-party national performance testing centers of China. These samples were collected precisely between the reciprocal third and reciprocal fourth ribs of the longissimus dorsi. A, Shear force. B, Meat color score. C, $\mathrm{pH} 1$ ( $\mathrm{pH}$ of the lean meat $45 \mathrm{~min}$ after slaughter). D, Drip loss: the percentage of water loss of the lean meat after hanging for $24 \mathrm{~h} . * *, P<0.05$; **, $P<0.01$; ***, $P<0.001$; not significant, $P>0.05$, not labeled.

For the future application of MSTN-editing in pig breeding, the hindlimb weakness in commercial newborn $M_{S T N^{-1}}$ pigs is a serious bottleneck. These piglets are homozygous for a deletion in the last exon of MSTN, exon 3, and a highly lean strain of LW pigs with a natural mutation in this exon was recently reported (Matika et al., 2019). Our data enable the isolation of the effects from both breeds and the editing position. The same phenotype was confirmed in our MSTNedited LW pigs, which show a frameshift mutation in exon 3. In contrast, our $\mathrm{MSTN}^{-1-} \mathrm{MS}$ pigs for a frameshift deletion in the same region avoided lameness. This suggests that there are certain dominant genes in the genome of these Chinese indigenous breeds that can rescue this phenotype. Such genes may be located in chromosomal regions that are hotspots for structural variations and mutations that confer many of the differences between Asian and European pig breeds (Ai et al., 2015; Groenen et al., 2012).

However, the Western breeds, such as LW and Landrace, with a frameshift mutation near the initiation codon of MSTN (in exon 1) were able to stand and move normally despite also having a muscular phenotype. Two separate studies of a tri-hybrid breed (Duroc $\times$ Landrace $\times \mathrm{LW}$ ) wherein exon 1 was edited using different molecular tools support this phenomenon. Both editing sites reported in these studies result in a frameshift mutation close to the N-terminus of the protein, leading to the translation of a peptide with very low molecular weight compared with the pigs with exon 3-edited sites (Cyranoski, 2015; Tanihara et al., 2016). The molecular mechanism of the exon 3 -edited protein is clear, sharing a similar pathogenesis mechanism to human inclusion body myopathy (Wójcik et al., 2005). The fundamental issue here is that the exon 3-edited protein cannot dimerize and therefore, cannot be exported normally from cells, leading to the generation of inclusion bodies. One notable difference from human inclusion body myopathy is that MSTN proteins aberrantly accumulated in elderly patients do not properly fold in the endoplasmic reticulum in a timely manner (Wójcik et al., 2005). It is notable that no natural homozygous mutations of MSTN have been reported to date in the clinical literature (Schuelke et al., 2004). Importantly, our demonstration that it is possible to overcome hindlimb weakness clears the way for future applications of MSTNediting in pigs, including LW pigs.

Perceived limitations of the future application of MSTNediting mainly involve uncertainty regarding feed conversion and reproduction. The FCR directly determines the cost of raising pigs (Fowler et al., 1976; Kanis et al., 2005). In this study, we confirmed that reduced exercise-related HP in the $M S T N$-edited pigs compensated for the increased heat gain resulting from MSTN gene inactivation, which ultimately 
A

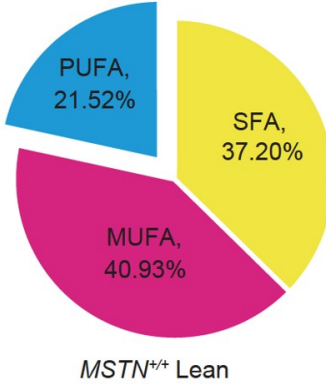

B

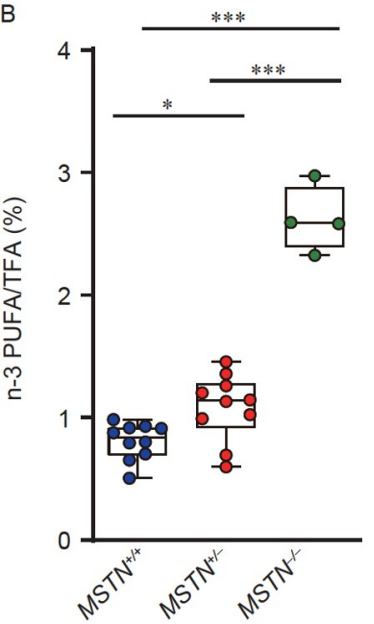

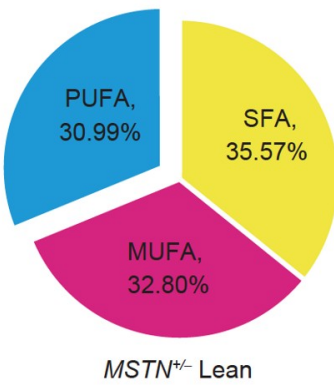
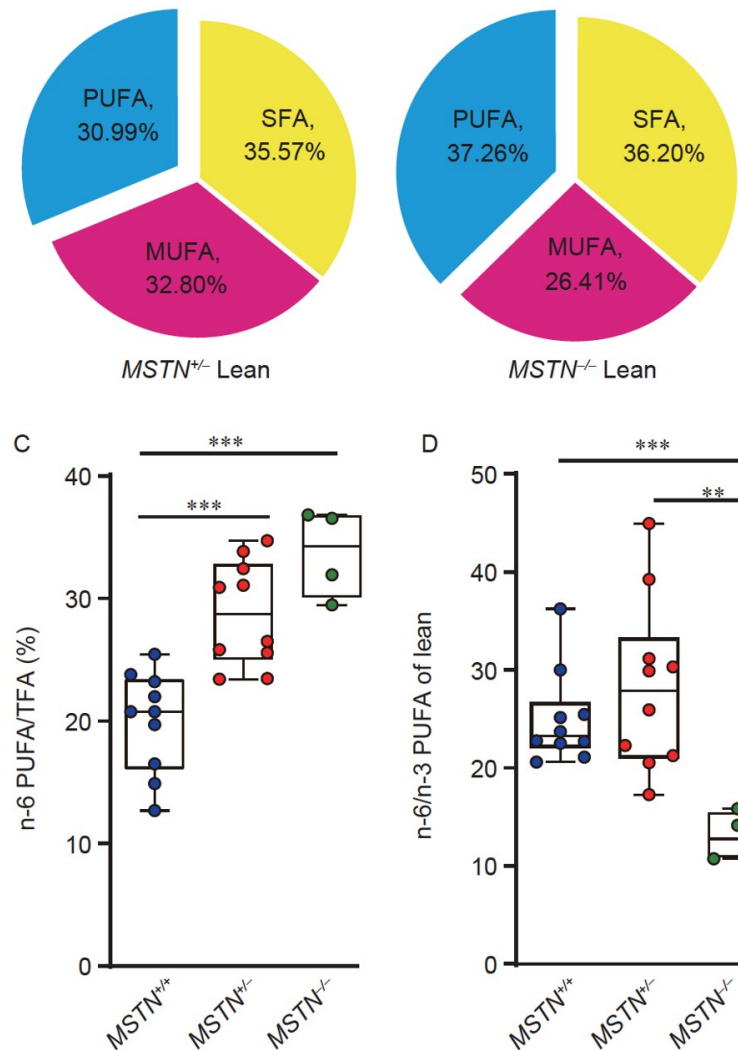

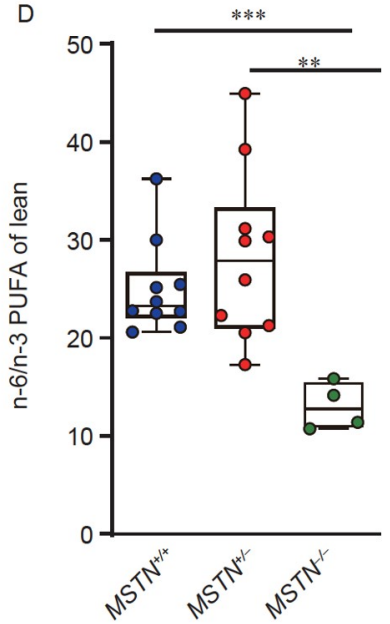

Figure 7 Increased healthy fatty acid composition of $M S T N$-edited lean meat. A, $M S T N$-editing increased the level of unsaturated fatty acids in lean meat in LW pigs $\left(M S T N^{-1-}, N=4 ; M S T N^{+-}, N=10\right.$; and WT, $\left.N=10\right)$. SFA (saturated fatty acid), MUFA (monounsaturated fatty acid), and PUFA (polyunsaturated fatty acid). B, n-3 PUFA content. C, n-6 PUFA content. D, n-6/n-3, lean PUFA ratio. * $P<0.05$; **, $P<0.01$; ${ }^{* * *}, P<0.001$; not significant, $P>0.05$, not labeled.

resulted in there being no difference in the FCR of MSTNedited and WT pigs. If reduced activity is associated with decreased aggression, this inactivity may also be beneficial for animal welfare. In addition, no dystocia was observed in the MSTN-edited sows, even in the first pregnancy.

Although some studies have confirmed that inactivation of $M S T N$ can greatly improve meat production performance in pigs, the basis of this improvement remains unclear. We found two distinct modes of improvement of the lean meat rate in $M S T N$-edited MS and LW pigs, revealing the different utilization strategies among breeds. Only intra-breed improvement by $M S T N$-editing was able to overcome the restrictions of small population size to achieve breeding goals. $M S T N^{-1-}$ pigs in Chinese breeds have exceeded lean meat rates of Western commercial breeds (65\%-70\%). Compared with the $\mathrm{MSTN}^{-1}$ pigs, the extent of lean meat rate improvement in the $M S T N^{+/-}$MS pigs was minimal, suggesting that $\mathrm{MSTN}^{-1-}$ pigs are most suitable for improving Chinese breeds.

In contrast, Western commercial breeds and supporting lines have experienced hybridization and high-intensity selection pressure and have a relatively high lean meat rate. It is thus extremely challenging to further improve the lean meat rate of such breeds. $M S T N^{+/-}$pigs have an increased lean meat rate of $\sim 8 \%$; the $M S T N^{-1}$ boars would be best utilized as the terminal paternal source for mating with WT sows as the resulting $M S T N^{+/-}$pigs would be highly suitable for commercial pig production. Based on our results, we deduced that, at the same carcass weight $(\sim 120 \mathrm{~kg})$, MSTNediting of commercial breeds can increase the lean meat yield by up to $\sim 4.5 \mathrm{~kg}$ per carcass, which should represent a remarkable increase in profits for large-scale farms. The advantages of $M S T N$-edited pigs are more prominent at this critical historical juncture when conventional pig breeding has been shelved or slowed down by the serious decline in breeding pig stocks caused by the African swine fever since 2018 in China and the doubling of the global hungry population caused by the coronavirus disease 2019 (COVID-19) pandemic in 2020 (Food Security Information Network, 2020). Most importantly, although the ratio of feed-to-pork was not changed by $M S T N$-editing, improvement of the lean meat rate significantly increased the efficiency of feed conversion to animal protein in both Chinese breeds and commercial breeds, which is in line with the development of environmentally sustainable modern low-carbon animal husbandry.

One previous study reported a few meat quality traits for $M S T N$-edited MS pigs, but these data are still preliminary, 
and the changes in meat quality and nutrition traits that can result from $M S T N$-editing remain poorly understood. Whereas pork is typically classified as "red meat," our data actually show that $M S T N$-edited pork has characteristics, including higher protein, lower fat, and more delicate texture, and there is evidence that white meat is healthier for humans (Etemadi et al., 2017; Zheng et al., 2019). Furthermore, highlighting the potential nutritional benefits, our study indicates that $M S T N$-edited pork contains higher PUFA levels, which have long been known to contribute to preventing cardiovascular and cerebrovascular diseases (Sokoła-Wysoczańska et al., 2018), and a breed with PUFA-rich pork was developed (Pan et al., 2010; Ren et al., 2011). Thus, $M S T N$-edited pork represents a better choice of meat product with significant market potential.

In summary, our work demonstrated that hindlimb weakness in $\mathrm{MSTN}^{-1-}$ piglets can be overcome. Our detailed longterm production performance measurements confirm that $M S T N$-editing can substantially accelerate the breeding progress for Western commercial breeds and can also confer improvement of all Chinese local breeds, thus providing a potentially attractive, novel strategy for breeders and a new choice for consumers in the future commercial chain.

\section{MATERIALS AND METHODS}

\section{Isolation of porcine embryonic fibroblasts}

Porcine embryonic fibroblasts (PEFs) were isolated from male fetuses of MS and LW pigs ( Day 35) and cultured in Dulbecco's Modified Eagle's medium (Gibco, USA) supplemented with $15 \%$ fetal bovine serum at $37.5^{\circ} \mathrm{C}$ and $5 \%$ $\mathrm{CO}_{2}$ in a humidified incubator.

\section{Preparation of fibroblasts with exon 3 of MSTN edited by TALEN}

Plasmids used for TALEN were designed and constructed by CWBiotech (Taizhou, China). LW and MS PEF lines were transfected with TAL-MSTN-F1 and TAL-MSTN-R1 using Nucleofector (Amaxa, Germany), in accordance with the manufacturer's guidelines using the program T-016. After transfection, the PEFs were cultured at $30.0^{\circ} \mathrm{C}$ for $72 \mathrm{~h}$ and seeded at $\sim 200$ cells per 10 -cm culture dish. PCR and DNA sequencing were used to identify positive cell clones for gene editing using the primers Exon-3F (TTGCTACTATTAACTCTTCTTTCA) and Exon-3R (TATATTATTTGTTCTTTGCCATTA).

\section{sgRNA design and preparation of fibroblasts with exon 1 of MSTN edited using CRISPR/Cas9}

We designed two sgRNAs targeted toward exon 1 of MSTN for precise deletion (MSTN-sgRNA1: TGTTCTCATTCAGATCCACGG and MSTN-sgRNA2: GAAAATGTGGAAAAAGAGGGG; PAM sequences have been underlined). Next, the two sgRNAs were separately inserted into the px330-GFP and px330-RFP plasmids, and LW PEFs were transfected with the plasmids. After transfection, the cells with both GFP and RFP signals were enriched by FACS. The enriched cell clones were identified using PCR and DNA sequencing of the MSTN-edited pigs using the primers Exon1F (TGTCTCTCAGACAGTGCAGG) and Exon1R (GTGTTTTGTCTCCACATACATGC) for genotype identification. The positive cells were used as nuclear donor cells for somatic cell nuclear transfer.

\section{Somatic cell nuclear transfer and embryo transfer}

All of the MSTN-edited founders were generated using somatic cell nuclear transfer and embryo transfer using previously described methods (Dai et al., 2002).

\section{Identification of cloned piglets}

To identify the MSTN genotypes of the cloned pigs and their offspring, ear samples were collected from the cloned pigs to extract genomic DNA for PCR. The primers were the same as those used for identification of positive cell lines.

\section{MSTN CDS sequencing}

RNA from the biceps femoris of the $M S T N^{-1-}$ and WT pigs was extracted and reverse-transcribed into cDNA. Primers were designed to amplify the full-length CDS of mRNA: MSTN CDS F, ATGCAAAAACTGCAAATCTATGTTTATATTTACCTGTT and MSTN CDS R, TCATGAGCACCCACAGCGATC. Subsequently, PCR products were Sangersequenced.

\section{Real-time PCR}

Tissue samples were homogenized using a Tissue Lyser (QIAGEN, Germany) and total RNA were extracted using TRIzol reagent (Invitrogen, USA). PCR was performed in triplicate for each sample and quantified using an ABI 7500 real-time PCR instrument (Applied Biosystems, USA). The relative expression levels of genes were calculated using the $2^{-\Delta \Delta C t}$ method. The primer sequences are listed in Table S7 in Supporting Information.

\section{Histological analysis}

Tissues were fixed using 4\% paraformaldehyde, dehydrated overnight in $70 \%$ ethanol, and embedded in paraffin. Sections were stained with H\&E. Images were captured using a 
microscope (DS-RI1; Nikon, Japan).

\section{TEM}

Tissues were fixed in $2 \%(\mathrm{v} / \mathrm{v})$ glutaraldehyde in $100 \mathrm{mmol} \mathrm{L}^{-1}$ phosphate buffer ( $\left.\mathrm{pH} 7.2\right)$ for $12 \mathrm{~h}$ at $4^{\circ} \mathrm{C}$. The samples were fixed using $1 \%$ osmium tetroxide, dehydrated using ascending gradations of ethanol, and embedded in fresh epoxy resin 618. Ultrathin sections were cut and stained with lead citrate before being examined via TEM (Philips CM-120 TEM, Philips, Netherlands).

\section{Western blotting}

The biceps femoris of MSTN-edited and WT piglets were lysed in lysis buffer. The samples were treated separately with nonreducing or reducing loading buffer for western blotting. The proteins were detected using antibodies against MSTN (Abcam, USA), Phospho-eIF2 $\alpha$ (Ser51; CST, USA), and eIF2 $\alpha$ (D7D3; CST).

\section{Propagation, establishment of founder breeding popu- lations, and molecular genetic analysis}

Adopting the linebreeding method, the LW and MS male founders were regarded as the ancestors. We screened for MSTN gene mutations in the offspring of both LW and MS pigs by PCR and sequencing.

\section{Duration of physical activity}

A continuous video system was used to observe the level of physical activity of the MSTN-edited $\left(M S T N^{-1-}\right)$ and WT individuals without human interference. For quantification, $\operatorname{MSTN}^{-1-}$ MS piglets $(N=4)$ and their WT littermates $(N=4)$ with similar body weights and ages ( 2 months old) were fed in adjacent pigsties without interference except for regular feeding and without water restrictions. The average duration of running was recorded.

\section{Net energy testing}

Net energy testing was facilitated by the Ministry of Agriculture and Rural Affairs Feed Industry Center, China. These experiments, including the protocols, were performed in accordance with previous studies (Li et al., 2017; Zhang et al., 2014). In short, three $M S T N^{-/-}$MS pigs and three WT littermates were chosen at random. A standard diet was provided during the experiment and pigs were individually housed in metabolism crates for 7 days to adapt to their feed, metabolic rhythm, and environmental conditions. The pigs were fasted on day 8 . The test was started $30 \mathrm{~h}$ after the last meal. All conditions, including regular light and unrestricted water supplies, remained unchanged. The $\mathrm{O}_{2}$ consumption, $\mathrm{CO}_{2}$ production, and HP were measured for $8 \mathrm{~h}$ from 10:00 pm to 6:00 am, and the pigs were kept in the dark to minimize their physical activity.

\section{Production performance testing}

China has a systematic pig breeding system that includes national and provincial pig performance testing centers and national core breeding farms with field testing stations. The production performance of the MSTN-edited pigs and WT pigs was evaluated based on carcass characteristics and indicators of reproduction, growth, and meat quality. These were determined by third-party national performance testing centers, including the Breeding Swine Quality Supervision and Testing Center of the Ministry of Agriculture and Rural Affairs of the People's Republic of China (Wuhan and Chongqing), and the testing stations on Ninghe National Original Pig Farm. All testings followed the guidelines stipulated in the "Rules for performance testing of breeding pigs" document published by the Ministry of Agriculture and Rural Affairs of the People's Republic of China (NY/T 8222004). The FCRs of LW pigs (from 30 to $100 \mathrm{~kg}$ ) and MS pigs (from D90 to D180) were measured using a performance measurement station. Briefly, electronic ear tags were identified by sensors when pigs entered the station to feed. Feed intake was calculated and recorded when entering and exiting the station and the weight of the feed tank was recorded. The weights of the pigs were recorded during the feeding process. Incoming weight, feed consumption, and outgoing weight were recorded and used to calculate FCR.

\section{Protein and amino acid content testing}

Protein and amino acid contents were tested at the Beijing Institute of Nutrition Sources, and the tests were in accordance with the standard internal method of the national food safety standard of the People's Republic of China for the determination of amino acids in food (GB 5009.1242016). Dorsi longus from LW pigs $\left(4 \mathrm{MSTN}^{-/-}\right.$vs. $9 \mathrm{MSTN}^{+/}$ vs. $12 \mathrm{MSTN}^{+/+}$) were measured.

\section{Fatty acid content testing}

Fatty acid content was tested at the Beijing Institute of Nutrition Sources and tests were conducted in accordance with the standard internal method of the national food safety standard of the People's Republic of China for the determination of fatty acids in food (GB 5009.168-2016). Dorsi longus from LW pigs $\left(4 \mathrm{MSTN}^{-/-}\right.$vs. $10 \mathrm{MSTN}^{+/-}$vs. 10 $\left.\mathrm{MSTN}^{+/+}\right)$and subcutaneous fat $\left(10 \mathrm{MSTN}^{+/-}\right.$vs. $9 \mathrm{MSTN}^{+/+}$; $M_{S T N}{ }^{-1}$ subcutaneous fat was too thin to collect) samples were measured. 


\section{Statistical analysis}

Data from each of the two groups of pigs were compared using unpaired $t$-test for nonnormal distribution. The significance levels were set at $0.05,0.01$, and 0.001 indicated by $*, * *$, and $* * *$, respectively. The data were analyzed with GraphPad Prism 8 for Windows (GraphPad Software).

Compliance and ethics The author(s) declare that they have no conflict of interest. All animal experiments described in this study were reviewed and approved by the Animal Care and Use Committee of the Institute of Animal Sciences, Chinese Academy of Agricultural Sciences. All experiments were performed in accordance with the approved guidelines for animal care and management (IAS2012-11).

\begin{abstract}
Acknowledgements This work was supported by the National Major Transgenic Breeding Project (2008ZX08006-003, 2011ZX08006-003, 2013ZX08006-003, 2014ZX08006-003, and 2016ZX08006-001), the National Key Basic Research Development Plan (2015CB943100), the Key Projects of the National Natural Science Foundation of China (30830080 and 31330074), and China Postdoctoral Foundation Project (2018M631648). We thank all researchers involved in these projects for their help and valuable suggestions. We would like to thank Prof. Xijun Yin and his research team at Yanbian University, and Prof. Yaofeng Zhao and his research team at China Agricultural University for valuable advice on this experiment. We also thank Ninghe National Original Pig Farm and the Breeding Swine Quality Supervision and Testing Center of the Ministry of Agriculture and Rural Affairs of the People's Republic of China (Wuhan and Chongqing) for performance testing. Finally, we thank Prof. Defa Li, Prof. Junjun Wang, Dr. Shuai Zhang, and Xiaoming Song of the China Agricultural University for useful discussions related to the design and execution of net energy experiments.
\end{abstract}

\section{References}

Ai, H., Fang, X., Yang, B., Huang, Z., Chen, H., Mao, L., Zhang, F., Zhang, L., Cui, L., He, W., et al. (2015). Adaptation and possible ancient interspecies introgression in pigs identified by whole-genome sequencing. Nat Genet 47, 217-225.

Boman, I.A., Klemetsdal, G., Nafstad, O., Blichfeldt, T., and Våge, D.I. (2010). Impact of two myostatin (MSTN) mutations on weight gain and lamb carcass classification in Norwegian White Sheep (Ovis aries). Genet Sel Evol 42, 4.

Cotton, T.R., Fischer, G., Wang, X., McCoy, J.C., Czepnik, M., Thompson, T.B., and Hyvönen, M. (2018). Structure of the human myostatin precursor and determinants of growth factor latency. EMBO J 37, 367383.

Cyranoski, D. (2015). Super-muscly pigs created by small genetic tweak. Nature 523, 13-14.

Dai, Y., Vaught, T.D., Boone, J., Chen, S.H., Phelps, C.J., Ball, S., Monahan, J.A., Jobst, P.M., McCreath, K.J., Lamborn, A.E., et al. (2002). Targeted disruption of the $\alpha 1,3$-galactosyltransferase gene in cloned pigs. Nat Biotechnol 20, 251-255.

Etemadi, A., Sinha, R., Ward, M.H., Graubard, B.I., Inoue-Choi, M., Dawsey, S.M., and Abnet, C.C. (2017). Mortality from different causes associated with meat, heme iron, nitrates, and nitrites in the NIH-AARP Diet and Health Study: population based cohort study. BMJ 357, j1957.

Food Security Information Network. (2020). Global Report on Food Crises 2020 September update: in times of COVID-19. Available from: URL: https://www.fsinplatform.org/sites/default/files/resources/files/ GRFC2020_September\%20Update_0.pdf.

Fowler, V.R., Bichard, M., and Pease, A. (1976). Objectives in pig breeding. Anim Sci 23, 365-387.

Groenen, M.A.M., Archibald, A.L., Uenishi, H., Tuggle, C.K., Takeuchi,
Y., Rothschild, M.F., Rogel-Gaillard, C., Park, C., Milan, D., Megens, H.J., et al. (2012). Analyses of pig genomes provide insight into porcine demography and evolution. Nature 491, 393-398.

Havlík, P., Valin, H., Herrero, M., Obersteiner, M., Schmid, E., Rufino, M. C., Mosnier, A., Thornton, P.K., Böttcher, H., Conant, R.T., et al. (2014). Climate change mitigation through livestock system transitions. Proc Natl Acad Sci USA 111, 3709-3714.

Joulia-Ekaza, D., and Cabello, G. (2006). Myostatin regulation of muscle development: molecular basis, natural mutations, physiopathological aspects. Exp Cell Res 312, 2401-2414.

Kambadur, R., Sharma, M., Smith, T.P.L., and Bass, J.J. (1997). Mutations in myostatin (GDF8) in double-muscled belgian blue and piedmontese cattle. Genome Res 7, 910-915.

Kang, J.D., Kim, S., Zhu, H.Y., Jin, L., Guo, Q., Li, X.C., Zhang, Y.C., Xing, X.X., Xuan, M.F., Zhang, G.L., et al. (2017). Generation of cloned adult muscular pigs with myostatin gene mutation by genetic engineering. RSC Adv 7, 12541-12549.

Kang, Q., Hu, Y., Zou, Y., Hu, W., Li, L., Chang, F., Li, Y., Lu, D., Sun, Z., Zhang, R., et al. (2014). Improving pig genetic resistance and muscle production through molecular biology. In: Proceedings, 10th World Congress of Genetics Applied to Livestock Production. Vancouver.

Kanis, E., De Greef, K.H., Hiemstra, A., and van Arendonk, J.A.M. (2005). Breeding for societally important traits in pigs. J Anim Sci 83, 948-957.

Keele, J.W., and Fahrenkrug, S.C. (2001). Optimum mating systems for the myostatin locus in cattle. J Anim Sci 79, 2016-2022.

Kolkman, I., Opsomer, G., Aerts, S., Hoflack, G., Laevens, H., and Lips, D. (2010). Analysis of body measurements of newborn purebred Belgian Blue calves. Animal 4, 661-671.

Lee, S.J., and McPherron, A.C. (1999). Myostatin and the control of skeletal muscle mass. Curr Opin Genets Dev 9, 604-607.

Li, Z.C., Li, P., Liu, D.W., Li, D.F., Wang, F.L., Su, Y.B., Zhu, Z.P., and Piao, X.S. (2017). Determination of the energy value of corn distillers dried grains with solubles containing different oil levels when fed to growing pigs. J Anim Physiol Anim Nutr 101, 339-348.

Matika, O., Robledo, D., Pong-Wong, R., Bishop, S.C., Riggio, V., Finlayson, H., Lowe, N.R., Hoste, A.E., Walling, G.A., Del-Pozo, J., et al. (2019). Balancing selection at a premature stop mutation in the myostatin gene underlies a recessive leg weakness syndrome in pigs. PLoS Genet 15, e1007759.

McPherron, A.C., Lawler, A.M., and Lee, S.J. (1997). Regulation of skeletal muscle mass in mice by a new TGF-beta superfamily member. Nature 387, 83-90.

McPherron, A.C., and Lee, S.J. (1997). Double muscling in cattle due to mutations in the myostatin gene. Proc Natl Acad Sci USA 94, 1245712461.

Merks, J.W.M., Mathur, P.K., and Knol, E.F. (2012). New phenotypes for new breeding goals in pigs. Animal 6, 535-543.

Mosher, D.S., Quignon, P., Bustamante, C.D., Sutter, N.B., Mellersh, C.S., Parker, H.G., and Ostrander, E.A. (2007). A mutation in the myostatin gene increases muscle mass and enhances racing performance in heterozygote dogs. PLoS Genet 3, e79.

Pan, D.K., Zhang, L., Zhou, Y.R., Feng, C., Long, C., Liu, X., Wan, R., Zhang, J., Lin, A.X., Dong, E.Q., et al. (2010). Efficient production of omega-3 fatty acid desaturase (sFat-1)-transgenic pigs by somatic cell nuclear transfer. Sci China Life Sci 53, 517-523.

Park, K.M., Kim, D.J., Paik, S.G., Kim, S.J., and Yeom, Y.I. (2006). Role of E2F1 in endoplasmic reticulum stress signaling. Mol Cells 21, 356-359.

Qian, L., Tang, M., Yang, J., Wang, Q., Cai, C., Jiang, S., Li, H., Jiang, K., Gao, P., Ma, D., et al. (2015). Targeted mutations in myostatin by zincfinger nucleases result in double-muscled phenotype in Meishan pigs. Sci Rep 5, 14435.

Ren, H., Zheng, X., Chen, H., and Li, K. (2011). Transgenic pigs carrying a synthesized fatty acid desaturase gene yield high level of co-3 PUFAs. Agric Sci China 10, 1603-1608.

Schuelke, M., Wagner, K.R., Stolz, L.E., Hübner, C., Riebel, T., Kömen, W., Braun, T., Tobin, J.F., and Lee, S.J. (2004). Myostatin mutation associated with gross muscle hypertrophy in a child. N Engl J Med 350, 
$2682-2688$.

Sokoła-Wysoczańska, E., Wysoczański, T., Wagner, J., Czyż, K., Bodkowski, R., Lochyński, S., and Patkowska-Sokoła, B. (2018). Polyunsaturated fatty acids and their potential therapeutic role in cardiovascular system disorders-A review. Nutrients 10, 1561.

Stinckens, A., Luyten, T., Bijttebier, J., Van den Maagdenberg, K., Dieltiens, D., Janssens, S., De Smet, S., Georges, M., and Buys, N. (2008). Characterization of the complete porcine MSTN gene and expression levels in pig breeds differing in muscularity. Anim Genets $39,586-596$.

Sutherland, C. (1985). Belgian blue cattle. Vet Record 117, 645-646.

Tanihara, F., Takemoto, T., Kitagawa, E., Rao, S., Do, L.T.K., Onishi, A., Yamashita, Y., Kosugi, C., Suzuki, H., Sembon, S., et al. (2016). Somatic cell reprogramming-free generation of genetically modified pigs. Sci Adv 2, e1600803.

Walker, R.G., Czepnik, M., Goebel, E.J., McCoy, J.C., Vujic, A., Cho, M., Oh, J., Aykul, S., Walton, K.L., Schang, G., et al. (2017). Structural basis for potency differences between GDF8 and GDF11. BMC Biol 15, 19.

Wang, K., Ouyang, H., Xie, Z., Yao, C., Guo, N., Li, M., Jiao, H., and Pang, D. (2015). Efficient generation of myostatin mutations in pigs using the CRISPR/Cas9 system. Sci Rep 5, 16623.
Wang, K., Tang, X., Xie, Z., Zou, X., Li, M., Yuan, H., Guo, N., Ouyang, H., Jiao, H., and Pang, D. (2017). CRISPR/Cas9-mediated knockout of myostatin in Chinese indigenous Erhualian pigs. Transgenic Res 26, 799-805.

Wójcik, S., Engel, W.K., McFerrin, J., and Askanas, V. (2005). Myostatin is increased and complexes with amyloid- $\beta$ within sporadic inclusionbody myositis muscle fibers. Acta Neuropathol 110, 173-177.

Yu, L.Z., Tang, H., Wang, J.Y., Wu, Y., Zou, L.L., Jiang, Y.L., Wu, C.X., and $\mathrm{Li}, \mathrm{N}$. (2007). Polymorphisms in the $5^{\prime}$ regulatory region of myostatin gene are associated with early growth traits in Yorkshire pigs. Sci China Ser C 50, 642-647.

Yu, Z., Li, Y., Meng, Q., Yuan, J., Zhao, Z., Li, W., Hu, X., Yan, B., Fan, B., Yu, S., et al. (2005). Comparative analysis of the pig BAC sequence involved in the regulation of myostatin gene. Sci China Ser C 48, 168 180.

Zhang, G.F., Liu, D.W., Wang, F.L., and Li, D.F. (2014). Estimation of the net energy requirements for maintenance in growing and finishing pigs. J Anim Sci 92, 2987-2995.

Zheng, Y., Li, Y., Satija, A., Pan, A., Sotos-Prieto, M., Rimm, E., Willett, W.C., and Hu, F.B. (2019). Association of changes in red meat consumption with total and cause specific mortality among US women and men: two prospective cohort studies. BMJ 365, 12110.

\section{SUPPORTING INFORMATION}

The supporting information is available online at https://doi.org/10.1007/s11427-020-1927-9. The supporting materials are published as submitted, without typesetting or editing. The responsibility for scientific accuracy and content remains entirely with the authors. 